\title{
Emergency medical dispatch services across Pan-Asian countries: a web-based survey
}

Shawn Chieh Loong Lee ${ }^{1,2^{*}}$ (D), Desmond Renhao Mao ${ }^{3}$, Yih Yng Ng${ }^{4}$, Benjamin Sieu-Hon Leong ${ }^{2}$, Jirapong Supasaovapak ${ }^{5}$, Faith Joan Gaerlan ${ }^{6}$, Do Ngoc Son ${ }^{7}$, Boon Yang Chia ${ }^{8}$, Sang Do Shin ${ }^{9}$, Chih-Hao Lin ${ }^{10}$, G. V. Ramana Rao ${ }^{11}$, Takahiro Hara ${ }^{12}$, Marcus Eng Hock Ong ${ }^{13,14}$ and For the PAROS Clinical Research Network

\begin{abstract}
Background: Dispatch services (DS's) form an integral part of emergency medical service (EMS) systems. The role of a dispatcher has also evolved into a crucial link in patient care delivery, particularly in dispatcher assisted cardiopulmonary resuscitation (DACPR) during out-of-hospital cardiac arrest (OHCA). Yet, there has been a paucity of research into the emerging area of dispatch science in Asia. This paper compares the characteristics of DS's, and state of implementation of DACPR within the Pan-Asian Resuscitation Outcomes (PAROS) network.
\end{abstract}

Methods: A cross-sectional descriptive survey addressing population characteristics, DS structures and levels of service, state of DACPR implementation (including protocols and quality improvement programs) among PAROS DS's.

Results: 9 DS's responded, representing a total of 23 dispatch centres from 9 countries that serve over 80 million people. Most PAROS DS's operate a tiered dispatch response, have implemented medical oversight, and tend to be staffed by dispatchers with a predominantly medical background. Almost all PAROS DS's have begun tracking key EMS indicators. $77.8 \%(n=7)$ of PAROS DS's have introduced DACPR. Of the DS's that have rolled out DACPR, 71.4\% $(n=5)$ provided instructions in over one language. All DS's that implemented DACPR and provided feedback to dispatchers offered feedback on missed OHCA recognition. The majority of DS's $(83.3 \% ; n=5)$ that offered DACPR and provided feedback to dispatchers also implemented corrective feedback, while $66.7 \%(n=4)$ offered positive feedback. Compression-only CPR was the standard instruction for PAROS DS's. OHCA recognition sensitivity varied widely in PAROS DS's, ranging from 32.6\% (95\% Cl: 29.9-35.5\%) to 79.2\% (95\% Cl: 72.9-84.4\%). Median time to first compression ranged from $120 \mathrm{~s}$ to $220 \mathrm{~s}$.

Conclusions: We found notable variations in characteristics and state of DACPR implementation between PAROS DS's.

These findings will lay the groundwork for future DS and DACPR studies in the PAROS network.

Keywords: Emergency medical services, Out-of-hospital cardiac arrest, Cardiopulmonary resuscitation, Asia-pacific, Public safety answering point

\section{Background}

Emergency medical dispatch is an emerging area of practice and research [1]. In the beginning, dispatchers in medical dispatch services (DS's) were typically laypeople with minimal to no training and took on a role akin to a telephone operator. The role of a dispatcher has since

\footnotetext{
* Correspondence: me@shawnlcl.com

'UNSW Medicine, University of New South Wales, Sydney, New South Wales 2033, Australia

${ }^{2}$ Emergency Medicine Department, National University Hospital, Singapore, Singapore

Full list of author information is available at the end of the article
}

evolved into a crucial link in delivering patient care in emergency medical service (EMS) systems, functioning as resource allocators, non-visual clinicians and gatekeepers who are able to implement lifesaving measures prior to responders arriving on scene [1,2], particularly in dispatcher assisted cardio-pulmonary resuscitation (DACPR) during out-of-hospital cardiac arrest (OHCA).

This evolution has occurred amidst a rapidly aging population in some countries and increased call volumes in the Asia-Pacific region, with emergency medical conditions, including OHCA, on the rise [3]. 


\section{Dispatch services in Asia and their role in out-of-hospital cardiac arrest}

EMS systems in Asia are heterogenous, and remain at different phases of maturity and development [4]. Asian countries' EMS setups are distinct from the AngloAmerican and Franco-German models, being relatively underdeveloped and with a comparatively short history spanning fewer than 20 years on average [5]. Globally, DS setups and operating procedures may vary greatly, with at least 6 different models identified in Europe alone [6].

OHCA survival rates in Asia remain relatively low [7]. The most significant modifiable element correlated with better neurological outcomes post-OHCA is the time from collapse to cardiopulmonary resuscitation (CPR) and defibrillation $[8,9]$. DACPR has been shown to raise survival and bystander CPR rates, and improve quality of life post-cardiac arrest [10].

This paper aims to describe the various DS's within the Pan Asian Resuscitation Outcomes Study (PAROS) Clinical Research Network, their practices and interventions, and the state of DACPR implementation within each DS, thereby laying a foundation for future research. PAROS was set up in 2009 with the aim of improving outcomes from pre-hospital emergency care across the Asia-Pacific, and currently spans 12 countries in the region.

\section{Methods}

We performed a cross-sectional, descriptive survey from July 2017 to March 2019. A web-based survey was disseminated to all medical directors of the DS's within the PAROS network.

Participants were given the option to respond via either a web-based survey system or email. A designated local principal investigator at each site was responsible for verifying and accurately entering the data. The local principal investigator also responded to data queries (Additional file 1).

\section{Definitions}

The Utstein recommendations were adopted alongside a unified taxonomy conceived by the PAROS network [11, 12]. Exclusion criteria for DACPR statistics were not finalized at the time of data collection as the revised 2017 American Heart Association quality improvement program recommendations were in the midst of being published when the survey was being disseminated $[13,14]$.

Key criteria for defining a DS in this study were (a) a common reporting agency or ministry, and; (b) a common operating framework and standard operating protocol, and; (c) a common service region (i.e. a city or state). Multiple dispatch centers (DCs), or physical callcenters that are responsible for taking emergency calls, may thus constitute one DS, so long as the three elements are present.
Call loads were calculated by obtaining the ratio of annual EMS transports to annual DS man hours, or the number of EMS transports activated per man hour.

\section{Survey tools}

A standardized survey form in English was used (Additional file 2). The survey was developed by PAROS investigators. Survey domains included study site's pre-hospital emergency care structure and characteristics, dispatcher credentials, process indicators of pre-arrival instructions and DACPR, characteristics of quality assurance program, population specific factors, and outcome measures.

\section{Results}

The survey was sent to 19 sites. Response rate to the survey was $47.4 \%(n=9)$, with $75 \%(n=9)$ of PAROS countries represented including India (1), Japan (1), Korea (1), Malaysia (1), Philippines (1), Singapore (1), Taiwan (1), Thailand (1) and Vietnam (1). These DS's manage a total of 23 dispatch centers and serve over 80 million people in Asia (Fig. 1). 66.7\% $(n=6)$ of service regions were urban. There was a large variation in call loads ranging from 0.21 to 7.66. Characteristics of each DS are listed in Table 1. Structure and staffing capabilities are listed in Table 2.

Quality improvement indicators measured for each DS are listed in Table 3. Most survey sites $(77.8 \% ; n=7)$ reviewed OHCA run sheets. $44.4 \%(n=4)$ shared a common OHCA patient registry between the ambulance service and receiving hospitals. Almost all survey sites $(88.9 \% ; n=8)$ tracked key EMS indicators, including the time from first contact to EMS dispatch, time from EMS dispatch to arrival at scene and time to arrival at hospital from the scene.

$77.8 \%(n=7)$ of survey sites have introduced DACPR. Specific DACPR characteristics are listed in Table 4. Of the DS's that have rolled out DACPR, $71.4 \%(n=5)$ provided instructions in over one language. In $28.6 \%(n=2)$ of DS's that have introduced DACPR, not all staff were trained to deliver DACPR instructions. 85.7\% $(n=6)$ of DS's that offered DACPR provided feedback for dispatchers. All DS's that implemented DACPR and provided feedback to dispatchers offered feedback on missed OHCA recognition. The majority of DS's (83.3\%; $n=5)$ that offered DACPR and provided feedback to dispatchers also implemented corrective feedback, while 66.7\% $(n=4)$ offered positive feedback.

DACPR statistics for DS's that have implemented DACPR are found in Table 5. OHCA recognition sensitivity ranged from $32.6 \%$ (95\% CI: $29.9-35.5 \%$ ) to $79.2 \%$ (95\% CI: 72.9-84.4\%). Median time to first compression ranged from $90 \mathrm{~s}$ to $220 \mathrm{~s}$. 


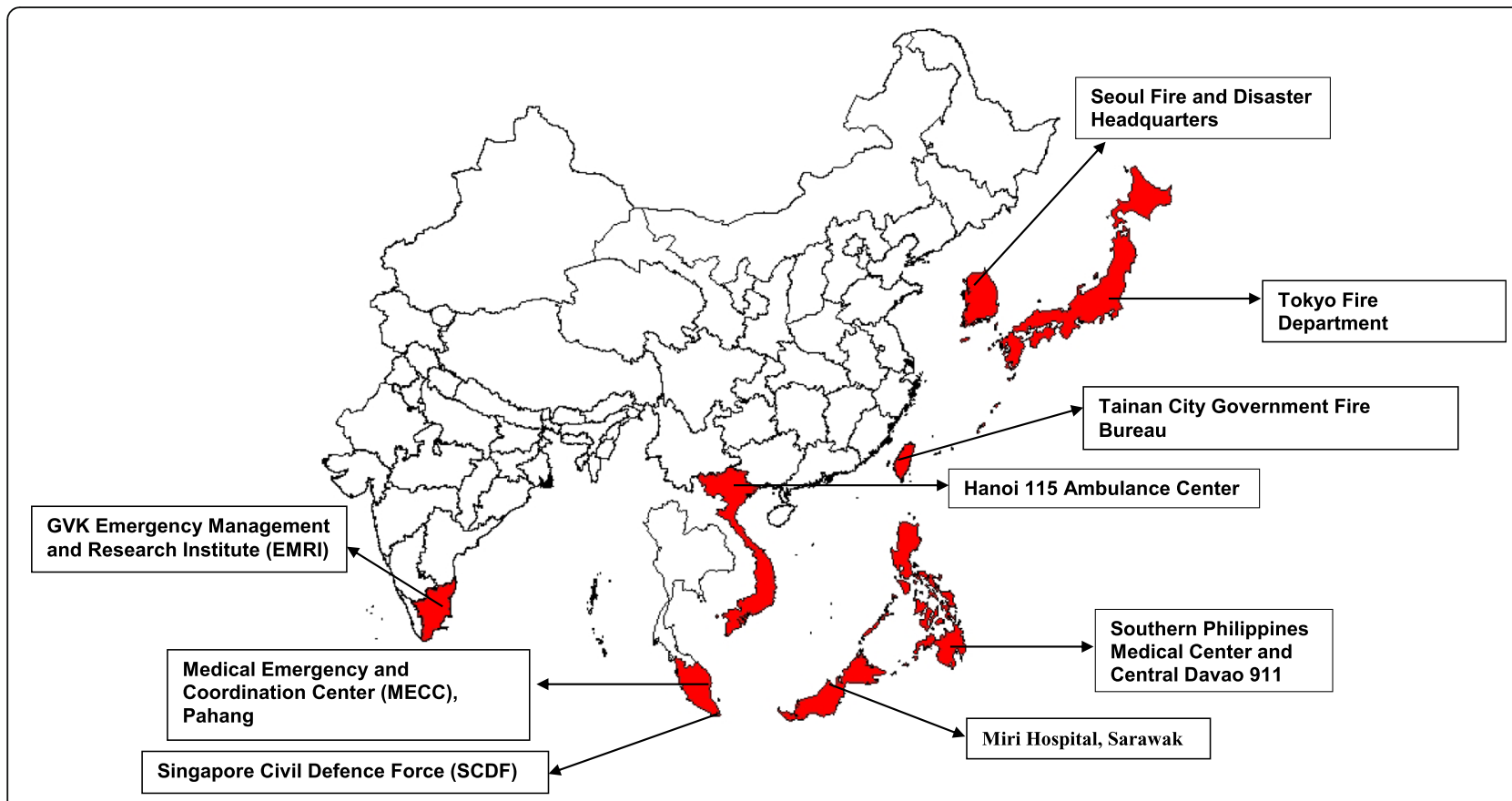

Fig. 1 Map of Respondents' Sites. The map was created using ArcGIS - ArcMap v10.0 (Esri, California, United States of America)

\section{Discussion}

Our study demonstrated many similarities and some variations in DS characteristics. Most PAROS DS's operated tiered response systems and were protocol-driven. Medical oversight was a clear feature in most DS's and dispatchers were predominantly healthcare providers, comprising EMTs (Emergency Medical Technicians), paramedics and nurses. DS's were tracking quality indicators for general EMS as well as DACPR domains.

With regard to DACPR, internally developed scripts were commonplace and had been translated to the local lingua franca. All DS's performed compression-only DACPR.

Table 1 Respondents' characteristics

\begin{tabular}{|c|c|c|c|c|c|c|c|c|c|}
\hline DS & Seoul & Hanoi & Tokyo & Miri & Davao & Tainan & Telangana & Singapore & Bangkok \\
\hline Country & Korea & Vietnam & Japan & Malaysia & Philippines & Taiwan & India & Singapore & Thailand \\
\hline Year Collected & 2015 & 2016 & 2015 & $2016^{a}$ & 2016 & $2013^{b}$ & 2016 & 2016 & 2017 \\
\hline Population & $9,471,871$ & $6,452,000$ & $13,491,000$ & 151,500 & $1,632,991$ & $1,840,257$ & $35,003,674$ & $5,612,253$ & $8,306,218$ \\
\hline Area $\left(\mathrm{km}^{2}\right)$ & 605.2 & 3329 & 2191 & 997.4 & 2444 & 2192 & 112,077 & 721.5 & 1569 \\
\hline Population Density $/ \mathrm{km}^{2}$ & 15,651 & 1938 & 6157 & 152 & 668 & 840 & 312 & 7779 & 5294 \\
\hline Urbanization & Urban & Urban & $\begin{array}{l}\text { Urban \& } \\
\text { Suburban }\end{array}$ & Rural & Urban & Urban & $\begin{array}{l}\text { Urban, Suburban } \\
\text { \& Rural }\end{array}$ & Urban & Urban \\
\hline Annual EMS Transports & 335,457 & 23,000 & $1,328,054$ & 1888 & 18,183 & 94,000 & 448,711 & 178,154 & 5000 \\
\hline Annual Total DS Man Hours & 43,800 & 26,280 & 700,800 & 8760 & 87,600 & 35,040 & 233,488 & 219,000 & 17,520 \\
\hline Transport to Man Hour Ratio & 7.66 & 0.86 & 1.90 & 0.22 & 0.21 & 2.68 & 1.92 & 0.81 & 0.29 \\
\hline $\begin{array}{l}\text { Paramedic-Confirmed OHCA } \\
\text { Calls }\end{array}$ & 4577 & 1000 & 23,477 & 135 & 10 & 1119 & 1709 & 2521 & 207 \\
\hline CPR trained (\%) & 10 & NA & 7 & 1 & 10 & NA & NA & 30.3 & NA \\
\hline AED trained (\%) & 7.5 & NA & 7 & 1 & 5 & NA & NA & 11.1 & NA \\
\hline Bystander CPR (\%) & 52 & NA & 39.9 & 5 & 6 & 25.8 & NA & 54.1 & 34 \\
\hline
\end{tabular}

DS Dispatch service, EMS Emergency medical service, OHCA Out of hospital cardiac arrest, AED Automated external defibrillator, Annual Total DS Man Hours Total number of man hours rostered annually by the Dispatch Service, Transport to Man Hour Ratio Number of EMS transports activated per man hour rostered by the Dispatch Service, Paramedic-Confirmed OHCA Calls Number of calls annually that were assessed by paramedics to be an OHCA

a Data was only available from April to December 2016

${ }^{b}$ Data was only available from 2013 due to site constraints 
Table 2 Dispatch service capabilities \& dispatcher qualifications

\begin{tabular}{|c|c|c|c|c|c|c|c|c|c|}
\hline$\overline{\mathrm{DS}}$ & Seoul & Hanoi & Tokyo & Miri & Davao & Tainan & Telangana & Singapore & Bangkok \\
\hline Number of DCs & 1 & 5 & 2 & 1 & 10 & 1 & 1 & 1 & 1 \\
\hline $\begin{array}{l}\text { DS } \\
\text { Configuration }\end{array}$ & Vertical & Horizontal & Horizontal & Vertical & Horizontal & Vertical & Vertical & Horizontal & Horizontal \\
\hline $\begin{array}{l}\text { Dispatch } \\
\text { System }\end{array}$ & $\begin{array}{l}\text { Protocol- } \\
\text { Driven }\end{array}$ & NA & Protocol-Driven & $\begin{array}{l}\text { Protocol- } \\
\text { Driven }\end{array}$ & $\begin{array}{l}\text { Guideline- } \\
\text { Driven }\end{array}$ & $\begin{array}{l}\text { Protocol- } \\
\text { Driven }\end{array}$ & Protocol-Driven & $\begin{array}{l}\text { Protocol- } \\
\text { Driven }\end{array}$ & $\begin{array}{l}\text { Guideline- } \\
\text { Driven }\end{array}$ \\
\hline $\begin{array}{l}\text { Highest Level } \\
\text { of Response }\end{array}$ & $B L S+A L S$ & BLS & $\begin{array}{l}\text { BLS + First } \\
\text { Responder }\end{array}$ & $B L S+A L S$ & $B L S+A L S$ & $\mathrm{BLS}+\mathrm{ALS}$ & $\mathrm{BLS}+\mathrm{ALS}$ & $B L S+A L S$ & $\mathrm{BLS}+\mathrm{ALS}$ \\
\hline $\begin{array}{l}\text { Tiered Dispatch } \\
\text { Response }\end{array}$ & Yes & Yes & Yes & No & No & No & Yes & Yes & Yes \\
\hline \multirow{4}{*}{$\begin{array}{l}\text { First Dispatch } \\
\text { Response }\end{array}$} & Ambulance & Ambulance & Ambulance & NA & NA & NA & Ambulance & Ambulance & Ambulance \\
\hline & \multirow[t]{3}{*}{$\begin{array}{l}\text { Fire } \\
\text { Appliance }\end{array}$} & & $\begin{array}{l}\text { Fire } \\
\text { Appliance }\end{array}$ & & & & & $\begin{array}{l}\text { Fire } \\
\text { Appliance }\end{array}$ & \\
\hline & & & Police Car & & & & & Motorcycle & Motorcycle \\
\hline & & & $\begin{array}{l}\text { Doctor } \\
\text { Ambulance }\end{array}$ & & & & & & \\
\hline DACPR & Yes & No & Yes & Yes & Yes & Yes & No & Yes & Yes \\
\hline $\begin{array}{l}\text { Other Pre-arrival } \\
\text { Instructions }\end{array}$ & Yes & No & Yes & Yes & Yes & Yes & No & Yes & Yes \\
\hline $\begin{array}{l}\text { Medical } \\
\text { Oversight }\end{array}$ & Yes & Yes & Yes & Yes & Yes & No & Yes & Yes & Yes \\
\hline $\begin{array}{l}\text { DS Standby } \\
\text { Physician }\end{array}$ & Yes & Yes & Yes & No & Yes & No & Yes & No & No \\
\hline $\begin{array}{l}\text { Predominant } \\
\text { Vocation }\end{array}$ & Paramedic & EMT & EMT & Paramedic & Nurse & Firefighter & $\begin{array}{l}\text { Emergency } \\
\text { Response Officer }\end{array}$ & Firefighter & Layperson \\
\hline $\begin{array}{l}\text { Minimum Entry } \\
\text { Qualification }\end{array}$ & $\begin{array}{l}\text { EMT } \\
\text { Intermediate }\end{array}$ & BCLS & EMT Basic & EMT Basic & EMT Basic & EMT Basic & Graduate & $\begin{array}{l}\text { First Aid + } \\
\text { CPR + AED }\end{array}$ & $\begin{array}{l}\text { First Aid + } \\
\text { CPR + AED }\end{array}$ \\
\hline $\begin{array}{l}\text { Regularly } \\
\text { Recertified }\end{array}$ & $\begin{array}{l}\text { EMT } \\
\text { Intermediate }\end{array}$ & BCLS & $\begin{array}{l}\text { EMT Basic } \\
\text { CPR } \\
\text { AED } \\
\text { First Aid }\end{array}$ & NA & $\begin{array}{l}\text { EMT Basic } \\
\text { CPR } \\
\text { AED }\end{array}$ & NA & First Aid & $\begin{array}{l}\text { BCLS } \\
\text { AED }\end{array}$ & CPR \\
\hline
\end{tabular}

DS Dispatch service, DC Dispatch Centre, Dispatch System Nature of call interrogation, BLS Basic life support, ALS Advanced life support, EMT Emergency medical technician, DACPR Dispatcher assisted cardio-pulmonary resuscitation, CPR Cardio-pulmonary resuscitation, BCLS Basic cardiac life support, AED Automated external defibrillator, Tiered Dispatch Response Dispatch response differs based on call severity, Other Pre-Arrival Instructions Instructions or guidance not involving DACPR provided by dispatcher to the individuals making the emergency call, Medical Oversight Physician supervision of the dispatch process, DS Standby Physician Physician(s) physically present in the dispatch center(s) to handle difficult calls, Minimum Entry Qualification Minimum qualification(s) required to be deployed as a dispatcher, Regularly Recertified Qualification(s) required to be regularly renewed

OHCA recognition sensitivity and compression start rates varied considerably between DS's.Compared to the previous survey published in 2012, Seoul, Tokyo and Singapore had transitioned from a single-tier to a tiered dispatch response system [5]. This could be a response to the aging populations they served requiring varying degrees of response [5], and overall increased call volumes. While most dispatchers had prior medical training, a protocol-driven dispatch system was predominant. In contrast to the stricter, protocoldriven dispatch systems that are algorithm-based, guideline-driven dispatch systems permit a more free-form and dynamic nature of communication [15]. Thus, the preference for protocol-driven dispatch systems may stem from concerns surrounding patient safety, as guideline-based dispatch systems require dispatchers to make more decisions, and are consequently at higher risk of poor outcomes [16].
Almost all study sites had medical oversight in place. This could be attributed to greater attention to prehospital work by stakeholders and changes in funding structures. We believe that this is beneficial for overall patient care as medical oversight has been shown to improve patient outcomes through direct influence over real-time medical decisions and formulation of dispatch guidelines and protocols [17].

Compared to 2015, measurement of quality indicators have been introduced in most DS's and EMS systems [4]. Recent years have seen the drive towards a 'quality-based' culture which is encouraged by both PAROS and the Global Resuscitation Alliance [18]. Between our study's DS's, there remains significant differences in which performance indicators are reported, thus limiting comparisons. Continued collaborative efforts will facilitate standardization.

The increase in number of DS's that have implemented DACPR compared to 2012 may also be attributed to 
Table 3 Dispatch service quality improvement indicators

\begin{tabular}{|c|c|c|c|c|c|c|c|c|c|}
\hline$\overline{D S}$ & Seoul & Hanoi & Tokyo & Miri & Davao & Tainan & Telangana & Singapore & Bangkok \\
\hline Review OHCA Run Sheets & Yes & No & Yes & Yes & Yes & Yes & No & Yes & Yes \\
\hline \multicolumn{10}{|l|}{$\begin{array}{l}\text { Sharing of OHCA Patient Data } \\
\text { Between DS and: }\end{array}$} \\
\hline AS & Yes & No & Yes & No & No & No & Yes & Yes & Yes \\
\hline $\mathrm{RH}$ & Yes & No & Yes & Yes & No & No & No & No & No \\
\hline AS \& RH in Common Registry & No & No & Yes & No & Yes & Yes & No & No & Yes \\
\hline EMS KPIs Measured & Yes & No & Yes & Yes & Yes & Yes & Yes & Yes & Yes \\
\hline First contact to EMS dispatch & Yes & NA & Yes & Yes & Yes & Yes & Yes & Yes & No \\
\hline EMS dispatch to arrival at scene & Yes & NA & Yes & Yes & Yes & Yes & Yes & Yes & Yes \\
\hline Arrival at hospital from scene & Yes & NA & Yes & Yes & Yes & Yes & Yes & Yes & Yes \\
\hline OHCA KPIs Measured & Yes & No & Yes & Yes & Yes & Yes & No & Yes & Yes \\
\hline EMS Time logs & Yes & NA & Yes & No & Yes & Yes & NA & Yes & Yes \\
\hline DACPR Recognition Rate & Yes & NA & Yes & Yes & No & Yes & NA & Yes & Yes \\
\hline Time to DACPR Recognition & Yes & NA & Yes & No & Yes & Yes & NA & Yes & No \\
\hline DACPR Start Rate & Yes & NA & Yes & Yes & No & Yes & NA & Yes & No \\
\hline Time to DACPR Start & Yes & NA & Yes & No & Yes & Yes & NA & Yes & No \\
\hline Compression start rate & Yes & NA & No & Yes & Yes & Yes & NA & Yes & Yes \\
\hline Time to first compression & Yes & NA & No & No & No & Yes & NA & Yes & Yes \\
\hline Barriers to recognition & Yes & NA & Yes & Yes & No & Yes & NA & Yes & No \\
\hline Barriers to compression & No & NA & No & Yes & No & Yes & NA & Yes & Yes \\
\hline Patient Outcomes Measured & No & No & Yes & No & Yes & Yes & No & Yes & Yes \\
\hline Pre-Hospital ROSC Rates & NA & NA & Yes & NA & Yes & Yes & NA & Yes & Yes \\
\hline Hospital admission rate & NA & NA & No & NA & No & Yes & NA & Yes & Yes \\
\hline Survival Rate & NA & NA & No & NA & Yes & Yes & NA & Yes & Yes \\
\hline Rate of good functional recovery & NA & NA & No & NA & No & Yes & NA & Yes & Yes \\
\hline
\end{tabular}

EMS Emergency medical service, OHCA Out of hospital cardiac arrest, DS Dispatch service, DACPR Dispatcher assisted cardio-pulmonary resuscitation, AS Ambulance service, $R H$ Receiving hospital, KPI Key performance indicator, ROSC Return of spontaneous circulation

Phase 2 of the PAROS study [19]. This study involved the introduction of a bundle of care to the participating PAROS dispatch services that included the implementation of a DACPR protocol and training program. Notably, in Asia, where many countries are multilingual, DACPR should be available in more than one language as language barriers are known to delay recognition of $\mathrm{OHCA}$ and initiation of DACPR [20], and increase dispatch times [21]. Unfortunately, this increases the staffing requirement in an already resource-limited region. In light of the potential complex multilingual environments, the preference for internally-developed scripts may be due to the need for phrasing to be simple enough to translate on-the-fly [22]. Future DACPR scripts should therefore strive to utilize simple, unambiguous and easy-to-translate language to facilitate this, in the absence of DACPR scripts in the local language.

Most DS's surveyed provided feedback to dispatchers that was both positive and corrective. Only 2 DS's, Tainan and Singapore, gave dispatchers information on patient outcomes. While obtaining feedback entails a greater degree of information integration with receiving hospitals, we believe this cost is well worth the effort. Dispatcher competencies in delivering DACPR are known to be partially dependent on feedback of patient outcomes [23], and having an avenue to obtain such feedback may improve rates of DACPR.

Sensitivities of OHCA recognition by PAROS DS's that have introduced DACPR appear lower compared to Europe and American DS's [24]. This may be due to a heterogenous population resulting in a more complex multilingual environment, further complicated by a population with relatively lower health literacy as large swathes of Asia are still developing [25]. Moreover, differences in dispatch algorithms, instructions and protocols also exist [26], and DACPR remains a fairly recent introduction within the network.

\section{Strengths and limitations}

To our knowledge, this study is the first in-depth DS survey that sheds light on the current practices and 
Table 4 Dispatcher assisted cardiopulmonary resuscitation characteristics

\begin{tabular}{|c|c|c|c|c|c|c|c|}
\hline DS & Seoul & Tokyo & Miri & Davao & Tainan & Singapore & Bangkok \\
\hline Country & Korea & Japan & Malaysia & Philippines & Taiwan & Singapore & Thailand \\
\hline Year Introduced & 2011 & 1994 & 2013 & 2017 & 2013 & 2011 & 1995 \\
\hline Year Data Collected & 2015 & 2015 & 2016 & 2016 & 2013 & 2016 & 2017 \\
\hline DACPR in $>1$ Language & No & Yes & Yes & Yes & Yes & Yes & No \\
\hline Dispatch Staff Trained (\%) & 100 & 100 & 100 & 60 & 100 & 100 & 80 \\
\hline Script Source & $\begin{array}{l}\text { Internally } \\
\text { Developed }\end{array}$ & $\begin{array}{l}\text { Internally } \\
\text { Developed }\end{array}$ & $\begin{array}{l}\text { Commercially } \\
\text { Acquired }\end{array}$ & $\begin{array}{l}\text { Internally } \\
\text { Developed }\end{array}$ & $\begin{array}{l}\text { Internally } \\
\text { Developed }\end{array}$ & $\begin{array}{l}\text { Internally } \\
\text { Developed }\end{array}$ & $\begin{array}{l}\text { Internally } \\
\text { Developed }\end{array}$ \\
\hline Computer or Card-Based & Card Based & Computer Aided & Computer Aided & Computer Aided & Card Based & Computer Aided & Card Based \\
\hline DACPR Instructions & $\begin{array}{l}\text { Compression } \\
\text { Only }\end{array}$ & $\begin{array}{l}\text { Compression } \\
\text { Only }\end{array}$ & $\begin{array}{l}\text { Compression } \\
\text { Only }\end{array}$ & $\begin{array}{l}\text { Compression } \\
\text { Only }\end{array}$ & $\begin{array}{l}\text { Compression } \\
\text { Only }\end{array}$ & $\begin{array}{l}\text { Compression } \\
\text { Only }\end{array}$ & $\begin{array}{l}\text { Compression } \\
\text { Only }\end{array}$ \\
\hline $\begin{array}{l}30: 2 \text { CPR in Specific } \\
\text { Indications }\end{array}$ & No & Yes & Yes & Yes & Yes & Yes & No \\
\hline Lookout for AEDs & Yes & Yes & Yes & Yes & Yes & Yes & No \\
\hline DACPR Feedback & Yes & Yes & Yes & No & Yes & Yes & Yes \\
\hline OHCA Survivors & No & No & No & NA & Yes & Yes & No \\
\hline Positive Feedback & No & No & Yes & NA & Yes & Yes & Yes \\
\hline Corrective Feedback & Yes & No & Yes & NA & Yes & Yes & Yes \\
\hline Missed OHCA Recognition & Yes & Yes & Yes & NA & Yes & Yes & Yes \\
\hline
\end{tabular}

OHCA Out of hospital cardiac arrest, DS Dispatch service, DACPR Dispatcher assisted cardio-pulmonary resuscitation, CPR Cardio-pulmonary resuscitation, $A E D$ Automated external defibrillator

DACPR outcomes in Asian DS's; the cross-sectional nature of surveys is an important limitation and subject to recall bias. Different settings of DS's (e.g. urban \& rural) and the year of data reported (2013 to 2017) limit comparisons.

Some DS's have since introduced improvements that are not captured in this survey. For example, one site only recently started DACPR, and data reported in this survey may not have reflected the improvements that have been made.

While we attempted to adopt a standardized taxonomy, our data was collected based on self-reporting and is susceptible to variability in the interpretation of questions and data points. Comparisons in DACPR statistics are also limited as the exclusion criteria were not finalized at the time of data collection.
Furthermore, although this study has attempted to compare how much call load each DS comes under by comparing the annual number of transports as a proxy for annual number of calls, to the total number of man hours rostered annually between DS's. This does not consider the actual number of calls, as one call may have zero or multiple transports, variability in the length of calls, and how call volume varies with time of day.

There is a lack of consistent or universal metrics for assessing the call load and how it affects manpower requirements in DS's. While existing studies on call centers frequently utilize Erlang $\mathrm{B}$ and Erlang $\mathrm{C}$ formulae to determine optimal staffing requirements, there is a paucity of research on their use in the DS setting. These remain further avenues for research.

Table 5 Dispatcher assisted cardiopulmonary resuscitation statistics

\begin{tabular}{|c|c|c|c|c|c|c|c|}
\hline DS & Seoul & Tokyo & Miri & Davao & Tainan & Singapore & Bangkok \\
\hline Country & Korea & Japan & Malaysia & Philippines & Taiwan & Singapore & Thailand \\
\hline Year Data Collected & 2015 & 2015 & $2016^{a}$ & 2016 & $2013^{b}$ & 2016 & 2017 \\
\hline Paramedic-Confirmed OHCA Calls & 4577 & 23,477 & 135 & 10 & 1119 & 2521 & 207 \\
\hline DS recognized, n (\%) & $2587(56.5)$ & $12,615(53.7)$ & NA & NA & $365(32.6)$ & $1348(53.5)$ & $164(79.2)$ \\
\hline DS recognized \& compression started, n (\%) & $2175(84.1)$ & $8158(64.7)$ & 60 & NA & $43(11.8)$ & $1143(84.8)$ & $31(18.9)$ \\
\hline Median time to first compression (s) & 174 & NA & 120 & NA & 143 & 220 & NA \\
\hline
\end{tabular}

OHCA Out of hospital cardiac arrest, DS Dispatch service

a Data was only available from April to December 2016

b Data was only available from 2013 due to site constraints 


\section{Future developments}

Looking ahead, resource constraints may compel PAROS DS's to capitalize on advances in technology. The growing smartphone penetration rate in Asia presents a ripe opportunity for the introduction of mobilephone positioning systems that dispatch CPR-trained lay volunteers, such as GoodSAM and PulsePoint [27]. These efforts may increase bystander CPR rates and decrease time to first compression and defibrillation.

Additionally, this may be complemented by videoassisted dispatching, as has been trialed by GoodSAM [28]. While this implementation focused on the remote initial assessment of trauma, studies have shown that video-assisted dispatching may improve the quality of DACPR provided compared to the current, audioinstructed method [29].

The advent of artificial intelligence may also help DS's cope with the anticipated increases in demand. For example, Singapore is looking to deploy an artificial intelligence (AI) driven speech-to-text real-time transcription solution to help reduce the time spent on collecting and transcribing information [30]. This could help reduce staffing requirements as less time may be spent per call, and potentially be used for translating information on the fly. PAROS DS's are thus well primed to make use of these technologies to overcome their resource constraints and challenges.

\section{Conclusion}

This is the first large-scale, network-wide assessment focusing on dispatch service characteristics and the state of implementation of DACPR within PAROS. Much regional variation between DS's exists in terms of qualifications, QI measurements, DACPR implementation and outcome measures. These findings will lay the groundwork for future DS and DACPR studies.

\section{Supplementary information}

Supplementary information accompanies this paper at https://doi.org/10. 1186/s12873-019-0299-1.

Additional file 1. Site Principal Investigators.

Additional file 2. Standardized Survey Form.

\section{Abbreviations}

AED: Automated External Defibrillator; ALS: Advanced Life Support; BCLS: Basic Cardiac Life Support; BLS: Basic Life Support; CPR: Cardiopulmonary Resuscitation; DACPR: Dispatcher-Assisted Cardio-pulmonary Resuscitation; DC: Dispatch Center; DS: Dispatch Service; EMS: Emergency Medical Service; EMT: Emergency Medical Technician; KPI: Key Performance Indicator; OHCA: Out-of-hospital cardiac arrest; PAROS: Pan-Asian Resuscitation Outcomes Study; ROSC: Return of Spontaneous Circulation

\section{Acknowledgements}

The authors would like to thank Ms. Nur Shahidah from Department of Emergency Medicine, Singapore General Hospital, Singapore for her support on this project.
PAROS Clinical Research Network

Participating Site Investigators:

AK Sarah and MN Julina (Hospital Sungai Buloh, Selangor, Malaysia), GY Naroo (Rashid Hospital, Dubai, United Arab Emirates), T Yagdir (Dubai

Corporation for Ambulance Services, Dubai, United Arab Emirates), N Khunkhlai and P Khruekarnchana (Rajavithi Hospital, Bangkok, Thailand), A Monsomboon (Siriraj Hospital, Bangkok, Thailand), T Piyasuwankul (Prince of Songkla University, Hatyai, Thailand), T Nishiuchi (Kindai University Faculty of Medicine, Osaka, Japan), PCI Ko (National Taiwan University Hospital, National Taiwan University, Taipei, Taiwan), KJ Song (College of Medicine, Seoul National University, Seoul, Korea), KD Wong (Hospital Pulau Pinang, Pulau Pinang, Malaysia), LP Tham (KK Women's \& Children's Hospital, Singapore), N Doctor (Singapore General Hospital), SO Cheah (Ng Teng Fong General Hospital, Singapore), MYC Chia (Tan Tock Seng Hospital, Singapore), HN Gan and L Tiah (Changi General Hospital, Singapore)

\section{Authors' contributions}

YYN and DRM conceived the study objectives and methodology and prepared the study protocol. SCLL, BSHL, MEHO and DRM gave input to the design of the study. YYN, JS, FJG, DNS, BYC, SDS, CHL, GVRR, TH and MEHO assisted in preparation and administration of the study and data acquisition. All authors assisted in data clarification and writing and review of the article. SCLL was responsible for all statistical analyses. SCLL and DRM had full access to all the data in the study and take responsibility for the integrity of the data and the accuracy of the data analysis. All authors endorse the data and conclusions. SCLL and DRM take responsibility for the paper as a whole. All authors read and approved the final manuscript.

\section{Funding}

This study was supported by grants from National Medical Research Council, Clinician Scientist Awards, Singapore (NMRC/CSA/024/2010 and NMRC/CSA/ 0049/2013), Ministry of Health, Health Services Research Grant, Singapore (HSRG/0021/2012). The funders had no role in study design, data collection and analysis, decision to publish, or preparation of the manuscript.

Availability of data and materials

All data generated or analysed during this study are included in this published article and its supplementary information files.

\section{Ethics approval and consent to participate}

This study was reviewed and approved by the SingHealth Centralized Institutional Review Board (CIRB 2013/604/C) and National Healthcare Group Domain Specific Review Board (DSRB 2013/00929), with waiver of informed consent provided under PAROS Phase 2 (Implementation of DACPR protocol).

\section{Consent for publication}

Not applicable.

\section{Competing interests}

$\mathrm{MEHO}$ reports funding from the Zoll Medical Corporation for a study involving mechanical cardiopulmonary resuscitation devices; grants from the Laerdal Foundation, Laerdal Medical, and Ramsey Social Justice Foundation for funding of the Pan-Asian Resuscitation Outcomes Study; an advisory relationship with Global Healthcare SG, a commercial entity that manufactures cooling devices; and funding from Laerdal Medical on an observation program to their Community CPR Training Centre Research Program in Norway. The other co-authors report no conflicts of interest.

\section{Author details}

${ }^{1}$ UNSW Medicine, University of New South Wales, Sydney, New South Wales 2033, Australia. ²Emergency Medicine Department, National University Hospital, Singapore, Singapore. ${ }^{3}$ Department of Acute and Emergency Care, Khoo Teck Puat Hospital, Singapore, Singapore. ${ }^{4}$ Medical Department, Singapore Civil Defence Force, Singapore, Singapore. ${ }^{5}$ Narenthorn EMS Center, Rajavithi Hospital, Bangkok, Thailand. 'Southern Philippines Medical Center, Davao, Philippines. ${ }^{7}$ Bach Mai Hospital, Hanoi, Vietnam. ${ }^{8}$ Emergency and Trauma Department, Miri Hospital, Miri, Sarawak, Malaysia. ${ }^{9}$ Seoul National University College of Medicine, Seoul, South Korea. ${ }^{10}$ Department of Emergency Medicine, National Cheng Kung University Hospital, College of Medicine, National Cheng Kung University, Tainan, Taiwan. ${ }^{11}$ GVK Emergency 
Management and Research Institute (GVK EMRI), Secunderabad, Telangana, India. ${ }^{12}$ Graduate School of Emergency Medical System, Kokushikan University, Tokyo, Japan. ${ }^{13}$ Department of Emergency Medicine, Singapore General Hospital, Singapore, Singapore. ${ }^{14}$ Health Services and Systems Research, Duke-NUS Medical School, Singapore, Singapore.

Received: 3 November 2019 Accepted: 30 December 2019

Published online: 07 January 2020

\section{References}

1. Gardett I, Clawson JJ, Scott G, Barron T, Patterson B, Olola C. Past, present, and future of emergency dispatch research: a systematic literature review. Ann Emerg Dispatch Response. 2013;1(2):29-42 Available from: https:// www.aedrjournal.org/past-present-and-future-of-emergency-dispatchresearch-a-systematic-literature-review/. Cited 21 Feb 2019.

2. Barron T, Patterson B. Listen to the line. Ann Emerg Dispatch Response. 2013;1(1) Available from: https://www.aedrjournal.org/1623-2/. Cited 21 Feb 2019.

3. Goh LG. Future health issues and delivery needs of the elderly. Singapore Med J. 1997;38(10):418-21 Available from: http://www.smj.org.sg/sites/ default/files/3810/3810ra4.pdf. Cited 22 Feb 2019.

4. Rahman NH, Tanaka H, Do Shin S, Ng YY, Piyasuwankul T, Lin C-H, et al. Emergency medical services key performance measurement in Asian cities. Int J Emerg Med. 2015;8(1):12 Available from: https://intjem.biomedcentral. com/articles/10.1186/s12245-015-0062-7. Cited 25 Jan 2019.

5. Do Shin S, Hock Ong ME, Tanaka H, Ma MH-M, Nishiuchi T, Alsakaf O, et al, Comparison of emergency medical services systems across Pan-Asian countries: a web-based survey. Prehospital Emerg Care. 2012;16(4):477-96 Available from: http://www.tandfonline.com/doi/full/10.3109/10903127.2 012.695433. Cited 25 Jan 2019.

6. European Emergency Number Association. Public safety answering points global edition. 2018.

7. Berdowski J, Berg RA, Tijssen JGP, Koster RW. Global incidences of out-ofhospital cardiac arrest and survival rates: systematic review of 67 prospective studies. Theatr Res Int. 2010;81(11):1479-87 Available from: https://linkinghub.elsevier.com/retrieve/pii/S0300957210004326.

8. Hasselqvist-Ax I, Riva G, Herlitz J, Rosenqvist M, Hollenberg J, Nordberg P, et al. Early cardiopulmonary resuscitation in out-of-hospital cardiac arrest. N Engl J Med. 2015;372(24):2307-15 Available from: http://www.ncbi.nlm.nih. gov/pubmed/26061835. Cited 25 Jan 2019.

9. Bobrow BJ, Panczyk M. Time to compress the time to first compression. J Am Heart Assoc. 2018;7(9) Available from: http://www.ncbi.nlm.nih.gov/ pubmed/29703812. Cited 25 Jan 2019.

10. Stiell I, Nichol G, Wells G, De Maio V, Nesbitt L, Blackburn J, et al. Healthrelated quality of life is better for cardiac arrest survivors who received citizen cardiopulmonary resuscitation. Circulation. 2003;108(16):1939-44 Available from: http://www.ncbi.nlm.nih.gov/pubmed/14530198. Cited 25 Jan 2019.

11. Cummins RO, Chamberlain DA, Abramson NS, Allen M, Baskett PJ, Becker L, et al. Recommended guidelines for uniform reporting of data from out-ofhospital cardiac arrest: the Utstein style. A statement for health professionals from a task force of the American Heart Association, the European Resuscitation Council, the Heart and Stroke. Circulation. 1991;84(2):960-75 Available from: http://www.ncbi.nlm.nih.gov/pubmed/1860248. Cited 24 Feb 2019.

12. Singapore Clinical Research Institute. Pan-Asian resuscitation outcomes study source documents. 2019. Available from: http://www.scri.edu.sg/index. php/source-documents. Cited 3 Jun 2019.

13. Resuscitation Academy. The road to recognition and resuscitation: the role of telecommunicators and T-CPR QI in cardiac arrest survival. 2017.

14. American Heart Association. Telephone CPR (T-CPR) program recommendations and performance measures. 2017. Available from: https:// cpr.heart.org/AHAECC/CPRAndECC/ResuscitationScience/TelephoneCPR/ RecommendationsPerformanceMeasures/UCM_477526_Telephone-CPR-TCPR-Program-Recommendations-and-Performance-Measures.jsp. Cited 21 Jan 2019.

15. Ellensen EN, Wisborg T, Hunskaar S, Zakariassen E. Dispatch guideline adherence and response interval — a study of emergency medical calls in Norway. BMC Emerg Med. 2016;16(1):40 Available from: http:// bmcemergmed.biomedcentral.com/articles/10.1186/s12873-016-0105-2. Cited 3 Aug 2019.
16. Nicholl J, Coleman P, Parry G, Turner J, Dixon S. Emergency priority dispatch systems-a new era in the provision of ambulance services in the UK. Prehosp Immed Care. 1999;3:71-5.

17. van der Hoeven JG, de Koning J, van der Weyden PKC, Meinders AE. Improved outcome for patients with a cardiac arrest by supervision of the emergency medical services system. Neth J Med. 1995;46(3):123-30 Available from: https://www.sciencedirect.com/science/article/pii/03002 9779400106J. Cited 25 Feb 2019.

18. Global Resuscitation Alliance. Program 2: begin telephone-CPR with ongoing training and quality improvement. 2017.

19. Ong MEH, Do Shin S, Tanaka H, Ma MH-M, Nishiuchi T, Lee EJ, et al. Rationale, methodology, and implementation of a dispatcher-assisted cardiopulmonary resuscitation trial in the Asia-Pacific (Pan-Asian Resuscitation Outcomes Study Phase 2). Prehospital Emerg Care. 2015;19(1): 87-95 Available from: http://www.tandfonline.com/doi/full/10.3109/1090312 7.2014.942482. Cited 24 Feb 2019.

20. Bradley SM, Fahrenbruch CE, Meischke H, Allen J, Bloomingdale M, Rea TD Bystander CPR in out-of-hospital cardiac arrest: the role of limited English proficiency. Resuscitation. 2011;82(6):680-4 Available from: https://www. resuscitationjournal.com/article/S0300-9572(11)00114-6/pdf. Cited 24 Feb 2019.

21. Meischke HW, Calhoun RE, Yip M-P, Tu S-P, Painter IS. The effect of language barriers on dispatching EMS response. Prehospital Emerg Care. 2013;17(4):475-80 Available from: http://www.tandfonline.com/doi/full/10.31 09/10903127.2013.811565. Cited 24 Feb 2019.

22. Pek JH, de Korne DF, Hannawa AF, Leong BSH, Ng YY, Arulanandam S, et al. Dispatcher-assisted cardiopulmonary resuscitation for paediatric out-ofhospital cardiac arrest: a structured evaluation of communication issues using the SACCIA ${ }^{\circledast}$ safe communication typology. Resuscitation. 2019;139: 144-51 Available from: https://www.sciencedirect.com/science/article/pii/ S0300957219301236. Cited 5 Jun 2019.

23. Bång A, Ortgren P-O, Herlitz J, Währborg P. Dispatcher-assisted telephone CPR: a qualitative study exploring how dispatchers perceive their experiences. Resuscitation. 2002;53(2):135-51 Available from: https://www. ncbi.n/m.nih.gov/pubmed/12009217. Cited 27 Mar 2019.

24. Viereck S, Møller TP, Rothman JP, Folke F, Lippert FK. Recognition of out-ofhospital cardiac arrest during emergency calls - a systematic review of observational studies. Scand J Trauma Resusc Emerg Med. 2017;25(1):9 Available from: http://sjtrem.biomedcentral.com/articles/10.1186/s13049-01 7-0350-8. Cited 28 Feb 2019

25. Grosse RN, Auffrey C. Literacy and health status in developing countries Annu Rev Public Health. 1989;10(1):281-97 Available from: http://www. annualreviews.org/doi/10.1146/annurev.pu.10.050189.001433. Cited 4 Sept 2019

26. Lin C-H, Ng YY, Chiang W-C, Karim SA, Do Shin S, Tanaka H, et al. Variation of current protocols for managing out-of-hospital cardiac arrest in prehospital settings among Asian countries. J Formos Med Assoc. 2016; 115(8):628-38 Available from: http://www.ncbi.nlm.nih.gov/pubmed/265966 89. Cited 10 Oct 2019.

27. Smith CM, Wilson MH, Ghorbangholi A, Hartley-Sharpe C, Gwinnutt C, Dicker $B$, et al. The use of trained volunteers in the response to out-ofhospital cardiac arrest - the GoodSAM experience. Resuscitation. 2017;121: 123-6 Available from: https://www.sciencedirect.com/science/article/pii/S03 00957217306743. Cited 3 Jun 2019.

28. Ter Avest E, Lambert E, De Coverly R, Tucker H, Griggs J, Wilson MH, et al. Live video footage from scene to aid helicopter emergency medical service dispatch: a feasibility study. Scand J Trauma Resusc Emerg Med. 2019;27(1): 55.

29. Lin YY, Chiang WC, Hsieh MJ, Sun JT, Chang YC, Ma MHM. Quality of audioassisted versus video-assisted dispatcher-instructed bystander cardiopulmonary resuscitation: a systematic review and meta-analysis. Resuscitation. 2018;123:77-85.

30. Koh F. Singlish also can, for this Al call system: The Straits Times; 2019. Available from: www.straitstimes.com/singapore/singlish-also-can-for-this-aicall-system

\section{Publisher's Note}

Springer Nature remains neutral with regard to jurisdictional claims in published maps and institutional affiliations. 